\title{
Caracterización de las deficiencias de macronutrientes en plantas de cebollín (Allium schoenoprasum L.)
}

\section{Characterization of macronutrient deficiencies in chives (Allium schoenoprasum L.) plants}

DIEGO ANDRÉS BERNAL'1

LEIDY CAROLINA MORALES ${ }^{1}$

GERHARD FISCHER ${ }^{1,2}$

JAIRO CUERVO ${ }^{1}$

STANISLAV MAGNITSKIY'

\section{Plantación de cebollín en}

invernadero.

Foto: J. Cuervo

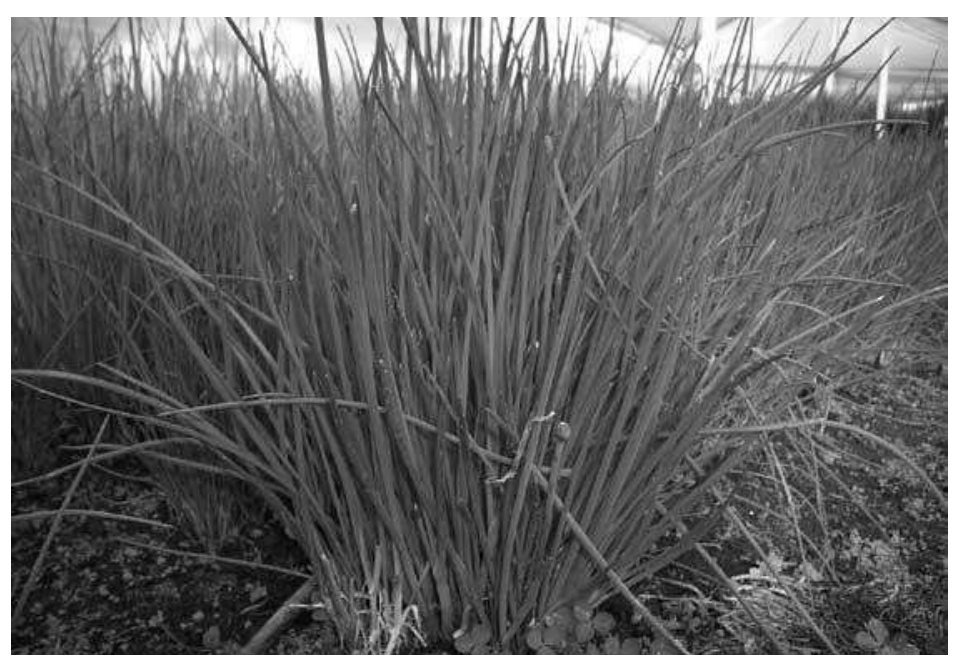

\section{RESUMEN}

El cebollín se encuentra dentro de la gama de plantas aromáticas exportadas desde Colombia al mercado internacional el cual es muy exigente en términos de volumen y calidad. Para caracterizar el efecto de deficiencias nutricionales de macroelementos en el crecimiento y desarrollo de las plantas, éstas fueron cultivadas bajo invernadero en Bogotá en materas de plástico $(2 \mathrm{~L})$ con sustrato arena cuarcítica durante dos ciclos de producción (primer corte a los 69 días después de la siembra, dds, y segundo a los 110 dds). Se evaluaron seis tratamientos; comparándolos con el testigo (T0) con fertilización completa, generados a partir de una solución de Hoagland y Arnon modificada: (T1) fertilización completa y N al 25\%; (T2) fertilización completa -P; (T3) fertilización completa -K; (T4) fertilización completa -Ca; (T5) fertilización completa-Mg y (T6) fertilización completa -S. Se evaluaron las variables número, diámetro y longitud de hojas y masa seca (MS) de hojas y raíces. La nutrición deficiente afectó especialmente el crecimiento y desarrollo del cebollín en el segundo ciclo de cultivo, en el cual la carencia de P redujo drásticamente el diámetro y la longitud foliar, la acumulación de MS en hoja y raíz, y el número de hojas en el primer ciclo. La concentración de $\mathrm{N}$ al 25\% disminuyó significativamente el diámetro y la longitud foliar durante el segundo ciclo de cultivo. La carencia de Mg aumentó el número y la biomasa de hojas en el segundo corte por el incremento de N, P, Ca y S en la masa seca foliar de este tratamiento. Los síntomas más comunes fueron la clorosis de las hojas con puntas amarillas hasta necróticas.

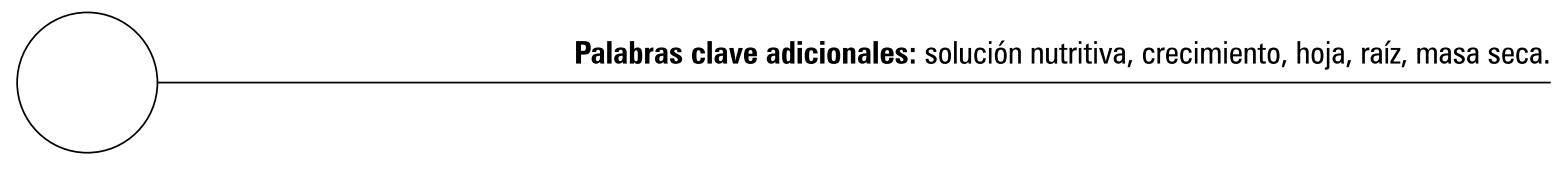

Departamento de Agronomía, Facultad de Agronomía, Universidad Nacional de Colombia, Bogotá (Colombia).

Autor para correspondencia. gfischer@unal.edu.co 


\section{ABSTRACT}

Chives plants belong to the group of aromatic plants exported from Colombia to the international market that requires products of high quality. In order to characterize the effect of deficiencies of macronutrients on the growth and development of chives plants, these were cultivated in greenhouse in Bogotá in $2 \mathrm{~L}$ plastic pots filled with quartz sand during two cycles of production (first harvest at 69 days and a second one at 110 days after planting). Six treatments were evaluated compared with the control (T0), which consisted in a complete fertilization using a modified solution of Hoagland and Arnon: (T1) complete fertilization with $\mathrm{N}$ at $25 \%$, (T2) complete fertilization $-\mathrm{P}$, (T3) complete fertilization $-\mathrm{K}$, (T4) complete fertilization -Ca, (T5) complete fertilization $-\mathrm{Mg}$, and (T6) complete fertilization $-\mathrm{S}$. Number, diameter and length of leaves and dry matter (DM) of leaves and roots were evaluated. The deficient nutrition affected, especially, the growth and development of chives during the second crop cycle, in which the lack of $\mathrm{P}$ drastically reduced diameter and length of leaves, accumulation of DM in leaves and roots, and also leaf number during the first cycle. The concentration of $25 \% \mathrm{~N}$ decreased significantly diameter and length of leaves during the second crop cycle. Deficiency of $\mathrm{Mg}$ increased the number and biomass of leaves in the second cycle by increasing concentrations of $\mathrm{N}, \mathrm{P}, \mathrm{Ca}$, and $\mathrm{S}$ in the leaf $\mathrm{DM}$ of this treatment. The most common visual symptoms of macronutrient deficiencies were leaf chlorosis and yellow to necrotic spots in leaf apices.

Additional key words: nutrient solution, growth, leaf, root, dry mass.

Fecha de recepción: 28-08-2008

Aprobado para publicación: 02-12-2008

INTRODUCCIÓN

El cebollín (Allium schoenoprasum L.) es una especie de hierba aromática, perteneciente a la familia Alliaceae. La planta posee un hábito de crecimiento perenne y presenta muchos ciclos de autorrenovación de sus estructuras vegetativas a través de los bulbillos (Delahaut y Newenhouse, 2003; Abello et al., 2006). Las hojas son el órgano de interés económico; estas son cilíndricas, tubulares y delgadas, con una longitud superior a $17 \mathrm{~cm}$, poseen un delicado aroma que las hace ser preferidas por los gourmet para destacar el sabor de diferentes platos, su consumo es generalmente en fresco. El cebollín se produce en climas fríos de Suramérica tropical, entre 2.000 y 2.800 msnm y bajo invernadero, aunque también se puede establecer a libre exposición, adaptándose a diferentes tipos de suelo (Barreño, 2006).
Investigaciones con especies aliáceas mostraron una asociación protectiva de estas contra el cáncer (Steinmetz y Potter, 1996), especialmente cáncer de estómago, debido a su contenido en compuestos como el dialil sulfido y alil metil trisulfido que inducen sistemas enzimáticos de detoxificación (You et al., 1989).

La ubicación geográfica en el trópico y su producción durante todo el año le han permitido a esta especie cobrar gran fuerza en las exportaciones colombianas con una participación del 8\% con respecto a las demás hierbas aromáticas culinarias vendidas en fresco. Los países principales a los cuales se exporta cebollín son Estados Unidos, Canadá, Inglaterra, Alemania y Holanda (Barreño y Clavijo, 2006). La gran exigencia de los mercados internacionales requiere de plantas 
de calidad, para lo cual es indispensable que la planta esté bien nutrida, esto permitirá el crecimiento y desarrollo adecuado de la raíz y de tejido foliar (Castro, 1998; Clavijo, 2006).

Los macronutrientes son elementos constituyentes de las biomoléculas estructurales $(\mathrm{N}, \mathrm{P}, \mathrm{S})$, tales como proteínas, lípidos o carbohidratos, o pueden actuar como osmolitos (K), sus concentraciones en los tejidos de las plantas pueden variar considerablemente dependiendo de la especie, la edad de la planta y el nivel de otros elementos (Bonilla, 2008). En cantidades óptimas, los nutrientes esenciales garantizan una buena producción y calidad de los cultivos siempre y cuando no se encuentren afectadas por otros factores; las plantas pueden afectarse por la falta de algún elemento, aun cuando los demás nutrientes estén presentes en cantidades adecuadas y disponibles para las plantas (Sabino et al., 2007). Las plantas responden a un aporte insuficiente de un elemento esencial, presentando síntomas característicos a esta deficiencia, muchos de estos son visuales, tal como la disminución en el crecimiento, clorosis, necrosis, entre otros (Martínez et al., 2009).

El nitrógeno forma parte de un gran número de compuestos orgánicos, incluidas hormonas de crecimiento (Wild y Jones, 1992); participa en la estructura de todas las proteínas, de los ácidos nucléicos, y además se encuentra como constituyente de las clorofilas y enzimas del grupo de los citrocromos y en varias coenzimas (Navarro y Navarro, 2000).

El fósforo forma enlaces anhídridos altos en energía, como es el caso del ATP y ADP, desempeñando, por tanto, un papel clave en la fotosíntesis, respiración y el metabolismo energético (Bonilla, 2008). Los fosfatos son, también, constituyentes de los ácidos nucléicos y fosfolípidos (Wild y Jones, 1992), los cuales son fundamentales en las membranas (Bonilla, 2008).
El potasio activa muchas enzimas que son esenciales en la fotosíntesis y la respiración (Salisbury y Ross, 2000) y actúa como osmorregulador, controlando en las células guarda la apertura de los estomas (Bennet, 1993). También participa en la producción de ATP, la síntesis de almidón y proteínas, y en el proceso de la fotosíntesis y el metabolismo de los carbohidratos (Navarro y Navarro, 2000).

Una de la principales funciones del Ca en la planta es formar parte de la estructura de la protopectina, como agente cementante para mantener las células unidas, estando localizado en la lámina media y en la pared primaria celular, además, participa en la regulación de la absorción de nitrógeno, la traslocación de los carbohidratos y proteínas, la neutralización de los ácidos orgánicos y la activación de algunas enzimas, tales como amilasa y fosfolipasa (Navarro y Navarro, 2000),

El $\mathrm{Mg}$ es un componente específico de la clorofila (porfirina magnésica) en la que un átomo de magnesio está ligado a cuatro anillos pirrólicos (Wild y Jones, 1992). Cumple un rol específico como activador de enzimas imvolucradas en la respiración, fotosíntesis y síntesis de $\mathrm{ADN}$ y ARN (Taiz y Zeiger, 2006). Actúa como cofactor de la mayor parte de las enzimas que intervienen en la fosforilación, y su importancia es grande en la transferencia de la energía (Wild y Jones, 1992).

Los compuestos orgánicos azufrados participan en la biosíntesis de lípidos, clorofilas, carotenos y ácidos orgánicos, aminoácidos sulfurados como cisteína, cistina y metionina y además, en la formación del acetilcoenzima A, eslabón básico de conexión entre glucólisis y el ciclo de Krebs (Navarro y Navarro, 2000), y la interacción del nitrógeno y azufre influye en la pungencia (aroma) de las plantas Allium (Liu et al., 2009), compuestos que pueden ser importantes como repelentes contra herbívoros (Öpik y Rolfe, 2005). 
El conocimiento de los síntomas de deficiencias minerales le permite determinar qué tipo de deficiencia nutricional puede tener el cultivo, establecer la mejor forma de aplicación y el momento más adecuado para fertilizar (Salisbury y Ross, 2000), permitiendo con ello generar modelos de nutrición mineral eficiente del cultivo (Le Bot et al., 1998). Si la concentración de un elemento nutriente esencial en el tejido vegetal está por debajo del nivel necesario para un óptimo crecimiento, indica que la planta es deficiente en ese elemento, y se produce así una alteración en la ruta metabólica en la que participa dicho elemento, afectando además otros procesos inmediatamente involucrados (Epstein y Bloom, 2005).

En Colombia no se registra un estudio enfocado hacia la caracterización de las deficiencias nutricionales en el cultivo de cebollín. Por tanto este trabajo tiene como objetivo caracterizar las deficiencias visuales de $\mathrm{N}, \mathrm{P}, \mathrm{K}, \mathrm{Ca}, \mathrm{Mg}$ y S a nivel foliar en plantas de cebollín estableciendo el efecto sobre diferentes variables de crecimiento, bajo una fertilización deficiente y, de esta manera, proporcionar herramientas de diagnóstico.

\section{MATERIALES Y MÉTODOS}

El trabajo se llevó a cabo en un invernadero de plástico de la Facultad de Agronomía de la Universidad Nacional de Colombia, Bogotá (2.556 $\mathrm{msnm})$, que presentó en el interior una temperatura promedio de $23^{\circ} \mathrm{C}$ y una humedad relativa promedio de $61 \%$. Durante el estudio incidieron 4,5 h brillo solar/día.

El trabajo inició con la siembra de las semillas de cebollín en materas de plástico, las cuales se llenaron con $2 \mathrm{~kg}$ de arena cuarcítica con un tamaño de grano de $0,5 \mathrm{~mm}$. Cada semilla fue sembrada a una profundidad de $0,5 \mathrm{~cm}$ con distancia de $3 \mathrm{~cm}$ (15 plantas/matera) de forma circular, las materas fueron ubicadas en una superficie plana de los bancos de propagación distanciadas entre sí a $30 \mathrm{~cm}$. Posterior a la siembra se cubrió con una lámina plástica de Vinipel (policloruro de vinilo) que permite un intercambio gaseoso entre las plantas y el ambiente, mantiene la humedad, la temperatura estable para acelerar la germinación de las semillas y bajar la pérdida de agua (Castro et al., 2003).

Se implementó un diseño de fertilización completamente al azar con seis tratamientos y cuatro repeticiones, comparados con el testigo (T0) fertilización completa: (T1) Fertilización completa y nitrógeno al 25\% (N 25\%), (T2): fertilización completa menos fósforo (-P), (T3): fertilización completa menos potasio (-K), (T4): fertilización completa menos calcio (-Ca), (T5): fertilización completa menos magnesio (-Mg) y (T6): fertilización completa menos azufre $(-S)$.

Para evaluar el efecto de los tratamientos, se utilizó la matriz de fertilización de Hoagland y Arnon modificada por H. Bolhuis (comunicación personal, 2008) (tabla 1), la cual fue mezclada para generar las soluciones en canecas de 20 L, estas fueron aplicadas vía fertirriego 1, 2 ó 3 veces por día con regadera dependiendo de las condiciones ambientales que se presentaran. Las semillas y plántulas se regaron con agua destilada durante 21 días después de siembra (dds) y para el día 22 se inició con la solución nutritiva que contenía todos los elementos hasta el día 35; con esta solución se buscó que las plantas tuvieran un buen desarrollo y fueran homogéneas al inicio de los tratamientos. Durante los tres días siguientes se regó solo con agua destilada con el fin de lavar el sustrato de los minerales que pudieran estar disponibles posteriormente para las plantas. Para el riego y preparación de las soluciones nutritivas se utilizó agua destilada; las fuentes de fertilizantes, suministrados por Brenntag Colombia S.A., Mosquera, fueron: Agrofeed Potasio Líquido, Potasa Cáustica, Ácido Fosfórico, MAP, Nitrato de Potasio, Nitrato de Amonio, Sulfato de Potasio y los micronutrientes. 
Tabla 1. Niveles de nutrientes ( $\mathrm{mg} \mathrm{L}^{-1}$ ) en solución nutritiva de Hoagland y Arnon, modificada según H. Bolhuis (comunicación personal, 2008) y aplicada diariamente.

\begin{tabular}{|c|c|c|c|c|c|c|c|}
\hline \multirow{2}{*}{ Nutriente } & TO & $\mathrm{T1}$ & T2 & T3 & T4 & T5 & T6 \\
\hline & Testigo & N 25\% & $-P$ & $-K$ & $-\mathrm{Ca}$ & $-\mathrm{Mg}$ & $-S$ \\
\hline $\mathrm{N}$ & 224,00 & 56,00 & 224,00 & 224,00 & 224,00 & 224,00 & 224,00 \\
\hline$P$ & 31,00 & 31,00 & - & 31,00 & 31,00 & 31,00 & 31,00 \\
\hline K & 235,00 & 235,00 & 235,00 & - & 235,00 & 235,00 & 235,00 \\
\hline $\mathrm{Ca}$ & 160,00 & 160,00 & 160,00 & 160,00 & & 160,00 & 160,00 \\
\hline $\mathrm{Mg}$ & 24,00 & 24,00 & 24,00 & 24,00 & 24,00 & - & 24,00 \\
\hline$S$ & 32,00 & 32,00 & 32,00 & 32,00 & 32,00 & 32,00 & - \\
\hline $\mathrm{Mn}$ & 0,11 & 0,11 & 0,11 & 0,11 & 0,11 & 0,11 & 0,11 \\
\hline $\mathrm{Zn}$ & 0,13 & 0,13 & 0,13 & 0,13 & 0,13 & 0,13 & 0,13 \\
\hline $\mathrm{Cu}$ & 0,03 & 0,03 & 0,03 & 0,03 & 0,03 & 0,03 & 0,03 \\
\hline $\mathrm{Fe}$ & 1,50 & 1,50 & 1,50 & 1,50 & 1,50 & 1,50 & 1,50 \\
\hline B & 0,27 & 0,27 & 0,27 & 0,27 & 0,27 & 0,27 & 0,27 \\
\hline Mo & 0,05 & 0,05 & 0,05 & 0,05 & 0,05 & 0,05 & 0,05 \\
\hline $\mathrm{Cl}$ & 140,00 & 140,00 & - & - & & - & - \\
\hline
\end{tabular}

Las evaluaciones se realizaron con una frecuencia de $7 \mathrm{~d}$ a partir del día 40 después de la siembra, durante dos ciclos de cultivo. Se evaluaron variables morfológicas, tal como longitud de hojas y de raíz (cm), diámetro medio de la hoja $(\mathrm{mm})$, número de las hojas, masa seca de hojas y raíz (g) y para cada uno de los tratamientos se realizó un análisis foliar a los 124 dds. El primer corte (cosecha) se realizó a los 69 dds y el segundo a los 110 dds.

Para el análisis de los datos se utilizó el paquete estadístico SAS ${ }^{\circledR} 9.1$ y en el análisis de varianza se compararon promedios mediante la prueba de Duncan $(P \leq 0,05)$ y contrastes ortogonales.

Los materiales de laboratorio utilizados fueron un pie de rey digital (marca Minutoyo-Vernier) para los diámetros de las hojas, balanza de precisión (0,001 g) marca Chyo Jupiter SDT-200 y estufa marca WTB binder para el secado del material vegetal a una temperatura de $70^{\circ} \mathrm{C}$ por $24 \mathrm{~h}$.

\section{RESULTADOS Y DISCUSIÓN}

\section{Contenido de los nutrientes en las hojas}

La concentración foliar de los diferentes elementos en las plantas testigo (tabla 2) mostró en orden decreciente $\mathrm{K}>\mathrm{N}>\mathrm{Ca}>\mathrm{Mg}>\mathrm{P}>\mathrm{S}$ lo que coincide, en los primeros tres elementos, con lo encontrado por Pôrto et al. (2006) en plantas de cebolla (hoja + bulbo) con $\mathrm{K}>\mathrm{N}>\mathrm{Ca}>\mathrm{S}>\mathrm{Mg}>\mathrm{P}$.

En las hojas de plantas deficientes se encontraron diferencias significativas $(P \leq 0,05)$ para la concentración de los seis elementos, especialmente para K, N, P, Ca y Mg (tabla 2). En el tratamiento $\mathrm{N} 25 \%$, la concentración de $\mathrm{N}$ foliar se redujo $29 \%$ comparado con el testigo. La tabla 2 indica que la reducción de $\mathrm{N}$ está relacionada con el incremento del nivel de $\mathrm{S}$, debido a que el S cumple funciones metabólicas similares en las cuales es importante el nitrógeno, de esta forma busca equilibrar el bajo contenido de este elemento (Sabino et al., 2007). Esto refleja la relación $\mathrm{N} / \mathrm{S}$ que 
en el testigo es 24,9 , mientras en plantas $\mathrm{N} 25 \%$ alcanza solamente 5,6 .

En el tratamiento -P se observó 55\% menos de la concentración de este elemento comparado con el testigo (tabla 2). La concentración óptima en la cual se debe encontrar el $\mathrm{P}$ en el tejido foliar del cebollín es de 0,3 a 0,5\% (Ramírez, 2006), muy similar a la óptima en hojas de cebolla $(0,25$ a $0,40 \%$ ) reportada por Bender (1993), lo que muestra el bajo contenido de este elemento en las hojas analizadas $(0,13 \%)$.

La concentración de K en las hojas del tratamiento $-\mathrm{K}$ fue $73,8 \%$ menor que el testigo (tabla 2 ), lo que confirma que los Allium son sumamente susceptibles a deficiencias de este elemento (Bender, 1993). En este tratamiento, las concentraciones de N, P, Ca y S muestran un incremento significativo, que puede ser debido a una serie de cambios químicos que generan una disminución en el contenido de almidón o la acumulación de compuestos solubles de nitrógeno. Marschner (2002) reporta que la falta de K suprime la síntesis de enzimas como la nitrato reductasa, lo que lleva a una acumulación de $\mathrm{N}$ en forma de nitrato en las hojas

La omisión de Ca en la solución nutritiva generó solamente una reducción del 13,7\% de la concentración de este elemento en la hoja que no fue significativa comparado con el testigo y que fue compensado por el aumento de otros nutrientes como el N, P y S en este órgano (tabla 2).

La deficiencia absoluta de $\mathrm{Mg}$ disminuyó la concentración de este elemento en las hojas en un $68,7 \%$ comparado con el testigo, pero se generó un aumento de los elementos N, P, Ca y $\mathrm{S}$ en este tratamiento (tabla 2), dentro de los cuales, el Ca puede parcialmente reemplazar el $\mathrm{Mg}$ en varias funciones (Bergmann, 1993).

La concentración de azufre muestra una disminución no significativa $(P \leq 0,05)$ de $15,0 \%$ en las hojas comparada con el testigo (tabla 2), generando incrementos en el $\mathrm{N}$ y $\mathrm{P}$ y reducción en el K. Alteraciones similares encontraron Alves et al. (2007) en plantas de Dimorphandra mollis.

\section{Síntomas foliares de la planta}

Las plantas de cebollín con deficiencia de $\mathrm{N}$ fueron más pequeñas (figura 1, tabla 4), con hojas más delgadas, cortas (tabla 4) y un color verde pálido (Bergmann, 1993), comparadas con el testigo. Se formó una clorosis basal en las hojas del primer ciclo, mientras que las maduras mostraron un mayor número de puntas (ápices) amarillas las cuales se necrosaron a medida que se acercó su senescencia. Los síntomas iniciaron en la parte inferior de las hojas maduras (Martínez

Tabla 2. Efecto de la deficiencia de $\mathrm{N}, \mathrm{P}, \mathrm{K}, \mathrm{Ca}, \mathrm{Mg}$ y S sobre la concentración de nutrientes en hojas de cebollín, a los 124 dds.

\begin{tabular}{|c|c|c|c|c|c|c|}
\hline \multirow{2}{*}{ Tratamiento } & $\mathbf{N}$ & $\mathbf{P}$ & K & $\mathrm{Ca}$ & Mg & S \\
\hline & \multicolumn{6}{|c|}{ Porcentaje en masa seca } \\
\hline Testigo & $3,98 \mathrm{c}$ & $0,29 \mathrm{~b}$ & 6,13 a & $0,51 \mathrm{~cd}$ & 0,32 a & $0,16 \mathrm{~b}$ \\
\hline N 25\% & $2,82 d$ & $0,18 \mathrm{c}$ & $4,98 \quad b$ & $1,19 b$ & $0,18 \mathrm{c}$ & $0,50 \mathrm{a}$ \\
\hline$-P$ & $4,15 \mathrm{c}$ & $0,13 \mathrm{c}$ & $3,88 \mathrm{c}$ & $1,10 \quad b c$ & $0,13 \mathrm{~cd}$ & 0,56 a \\
\hline$-K$ & 5,09 a & 0,43 a & $1,60 \mathrm{~d}$ & 1,90 a & $0,28 a b$ & 0,53 a \\
\hline$-\mathrm{Ca}$ & $4,86 a b$ & 0,52 a & $3,68 \mathrm{c}$ & $0,44 d$ & $0,25 b$ & 0,51 a \\
\hline$-\mathrm{Mg}$ & $4,86 a b$ & 0,41 a & $3,87 \mathrm{c}$ & $1,73 a b$ & $0,10 \mathrm{~d}$ & $0,55 \mathrm{a}$ \\
\hline$-S$ & $4,30 \mathrm{bc}$ & 0,44 a & 4,04 bc & $1,72 a b$ & $0,16 \mathrm{~cd}$ & $0,13 b$ \\
\hline
\end{tabular}

Promedios con letras distintas en la misma columna indican diferencia significativa según la prueba de Duncan $(P \leq 0,05)$. 
et al., 2009) donde se generó progresivamente una coloración amarilla en la medida que la deficiencia se acentuaba (Bender, 1993).

Las plantas de cebollín, carentes de $\mathrm{P}$, mostraron bajo crecimiento (Bender, 1993) y menor número de hojas (figura 1, tablas 3 y 4 ) que se agudizó, especialmente a partir de 113 dds, con hojas completamente delgadas, pequeñas y puntiagudas y en un $60 \%$ de estas se observaron puntas amarillas.

Las plantas con deficiencia de K expresaron hojas entorchadas hacía el ápice y un $50 \%$ de puntas amarillas, produciéndose necrosis del tejido foliar en dirección basipétala especialmente en las hojas más viejas, lo cual coincide con lo descrito por Bergmann (1993). Las hojas afectadas presentaron una coloración más oscura, como reporta Bender (1993) para cebolla (figura 1).
Las plantas con deficiencia de Ca no presentaron alteraciones en su crecimiento en el primer ciclo, sin embargo mostraron puntas amarillas en $80 \%$ de sus hojas. A los 92 dds se observaban hojas flácidas, débiles, con pérdida de turgencia y partidas. En el segundo ciclo empezaron a tomar colores más oscuras, con hojas quebradas, clorosis desde la base hacía el ápice, similar a los síntomas observados en plantas de uchuva (Martínez et al., 2009).

La deficiencia de $\mathrm{Mg}$ se inició a partir de 58 dds con la aparición de una línea blanca-clorótica en dirección vertical; a partir de 68 dds $30 \%$ de las hojas presentaron puntas amarillas que se transformaron después en necrósis conforme con la cebolla (Bender, 1993). Cuando 70\% de las hojas mostraron puntas amarillas perdieron su turgencia progresivamente y algunas se entorcharon (figura 1).

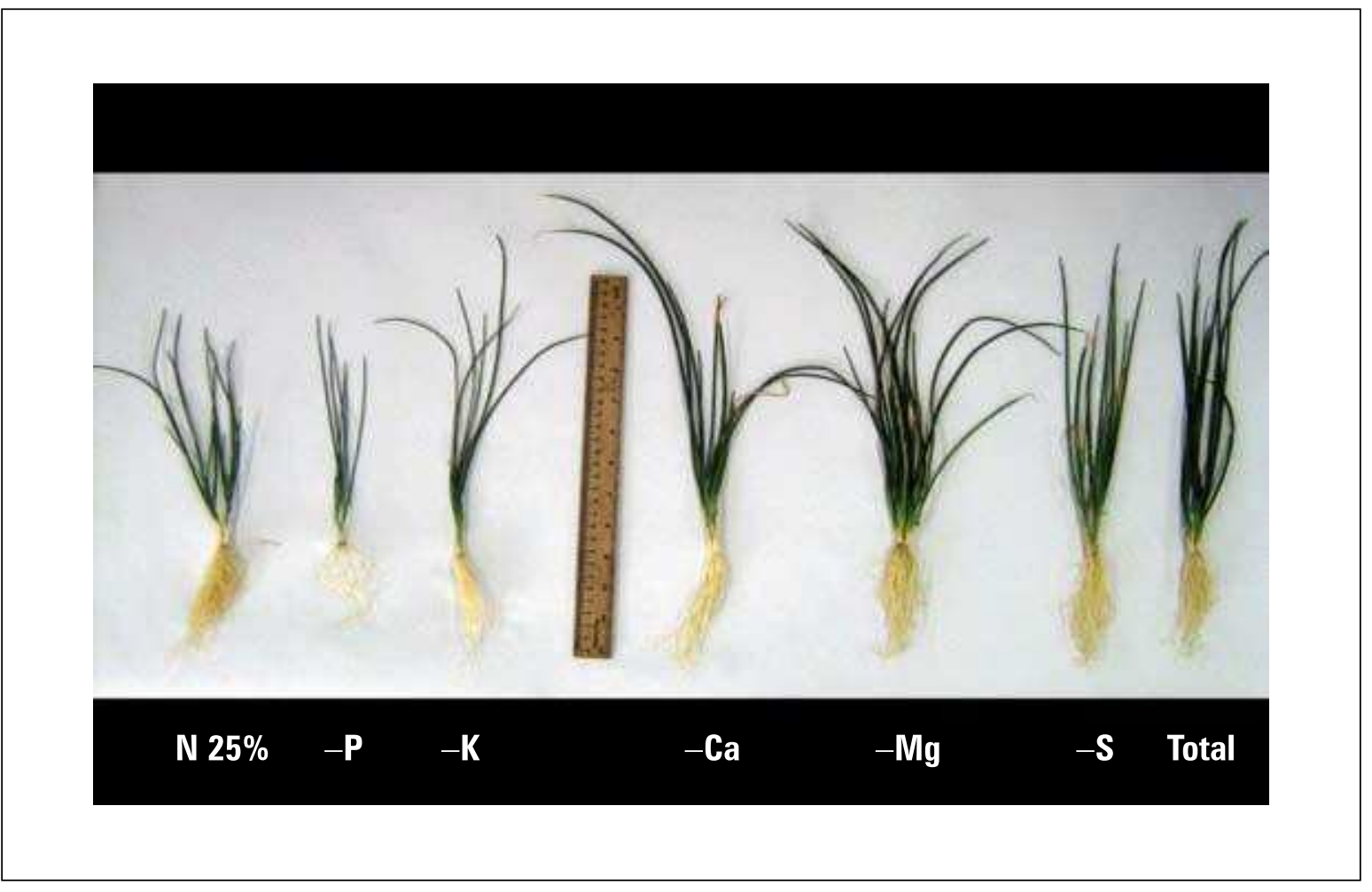

Figura 1. Desarrollo de plantas de cebollín con una nutrición ausente en $P, K, C a, M g$ y $\mathrm{S}$, así como $\mathrm{N}$ al $25 \%$, comparado con el testigo (Total) en el primer corte ( $69 \mathrm{dds})$. 
A final del primer ciclo, plantas deficientes de $\mathrm{S}$ presentaron algunos síntomas como clorosis, empezando en hojas nuevas (Bergmann, 1993) así como entorchamiento foliar, mientras en el segundo ciclo mostraron una clorosis basal que se agudizó a partir de 105 dds con un amarillamiento muy marcado y homogéneo y puntas amarillas, síntomas comunes por falta de S (Sabino et al., 2007).

\section{Número de hojas}

En el primer corte, la deficiencia de P disminuyó significativamente el número de hojas, comparado con el testigo (tabla 3), mientras la carencia de los demás elementos no afectó estadísticamente la emisión de hojas en cebollín.

El fosfato se redistribuye fácilmente de un órgano a otro acumulándose en las hojas jóvenes (Bonilla, 2008), por lo cual en el presente estudio se supone que la planta no contaba con una cantidad suficiente de este elemento lo que disminuyó la formación de hojas. Asimismo, la falta de ATP, ADP, AMP, los cuales son esenciales en el metabolismo energético, pudo haber causado una reducción en la emisión foliar (Salisbury y Ross, 2000; Jones, 1998).

Bender (1993) reporta que cebollas deficientes en azufre producen menos hojas; en cebollín este efecto no fue observado, debido, probablemente, a que la carencia de este elemento no fue total (tabla 2).

En el segundo corte se presentó un aumento del número de hojas en plantas deficientes del $\mathrm{Mg}$, comparado con las -P en la solución nutritiva (tabla 3). Un efecto que pudo haber sido causado por el aumento de N, P, Ca y S en concentraciones significativas en el tratamiento $-\mathrm{Mg}$, comparado con el testigo (tabla 2), teniendo en cuenta, por un lado, que elementos como el $\mathrm{Ca}$, además del Mn y Zn (tabla 1) pueden reemplazar el Mg en varias de sus funciones (Bergmann, 1993) y,

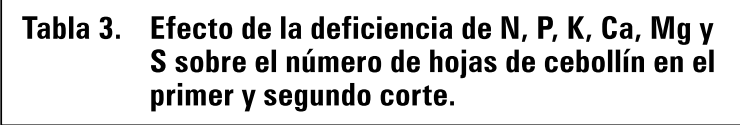

\begin{tabular}{|l|c|c|}
\hline \multirow{2}{*}{ Tratamiento } & \multicolumn{2}{|c|}{ Número de hojas } \\
\cline { 2 - 3 } & Primer corte & Segundo corte \\
\hline Testigo & $3,9 \mathrm{a}$ & $6,3 \mathrm{ab}$ \\
\hline $\mathrm{N} 25 \%$ & $3,3 \mathrm{ab}$ & $6,1 \mathrm{ab}$ \\
\hline$-\mathrm{P}$ & $3,1 \mathrm{~b}$ & $4,7 \mathrm{~b}$ \\
\hline$-\mathrm{K}$ & $3,3 \mathrm{ab}$ & $6,0 \mathrm{ab}$ \\
\hline$-\mathrm{Ca}$ & $3,4 \mathrm{ab}$ & $6,3 \mathrm{ab}$ \\
\hline$-\mathrm{Mg}$ & $3,0 \mathrm{ab}$ & $8,1 \mathrm{a}$ \\
\hline$-S$ & $3,5 \mathrm{ab}$ & $6,9 \mathrm{ab}$ \\
\hline
\end{tabular}

Promedios con letras distintas en la misma columna indican diferencia significativa según la prueba de Duncan $(P \leq 0,05)$.

por otro lado, la muy deficiente condición energética de plantas faltantes en $\mathrm{P}$.

\section{Diámetro y longitud de hojas}

Una de las características de las plantas de cebollín son sus hojas de forma cónica aguda, las cuales disminuyeron su diámetro significativamente $(P \leq 0,05)$ con respecto a su diámetro normal, lo que se observó en el primer corte en el tratamiento $-\mathrm{Mg}$ y al final del segundo ciclo en las plantas deficientes en Py K (tabla 4).

Se considera que al inicio del estudio las hojas de cebollín crecieron más lento en el tratamiento -Mg como reporta Bender (1993) para la cebolla, lo que podría haber afectado su aumento en diámetro. Hasta el segundo corte, las deficiencia de Py $\mathrm{N}$ se agudizaron afectando marcadamente el desarrollo foliar (tabla 4), siendo el P esencial en el metabolismo energético, condición indispensable para el crecimiento normal de la planta. Marschner (2002) describe que en las plantas deficientes en fósforo hay un retardo en el crecimiento de la parte aérea y se limita el proceso de fotosíntesis debido a alteraciones producidas en la concentración de clorofila. También en cebollas, Bender (1993) observó hojas más angostas por falta de nitrógeno. 
Tabla 4. Efecto de la deficiencia de N, P, K, Ca, Mg y S sobre el diámetro y la longitud de hojas de cebollín en el primer y segundo corte.

\begin{tabular}{|l|c|c|c|c|}
\hline \multirow{2}{*}{ Tratamiento } & \multicolumn{2}{|c|}{ Diámetro de hoja $(\mathrm{cm})$} & \multicolumn{2}{c|}{ Longitud de hoja $(\mathrm{cm})$} \\
\cline { 2 - 5 } & Primer corte & Segundo corte & Primer corte & Segundo corte \\
\hline Testigo & $1,67 \mathrm{ab}$ & $1,80 \mathrm{a}$ & $20,64 \mathrm{a}$ & $20,12 \mathrm{a}$ \\
\hline N 25\% & $1,82 \mathrm{a}$ & $1,34 \mathrm{~b}$ & $19,97 \mathrm{a}$ & $17,84 \mathrm{~b}$ \\
\hline -P & $1,54 \mathrm{ab}$ & $1,14 \mathrm{~b}$ & $18,46 \mathrm{a}$ & $14,29 \mathrm{~b}$ \\
\hline- K & $1,65 \mathrm{ab}$ & $1,50 \mathrm{ab}$ & $19,95 \mathrm{a}$ & $22,05 \mathrm{a}$ \\
\hline -Ca & $1,59 \mathrm{ab}$ & $1,53 \mathrm{ab}$ & $19,53 \mathrm{a}$ & $23,89 \mathrm{a}$ \\
\hline- Mg & $1,45 \mathrm{~b}$ & $1,65 \mathrm{ab}$ & $18,57 \mathrm{a}$ & $22,62 \mathrm{a}$ \\
\hline- S & $1,52 \mathrm{ab}$ & $1,53 \mathrm{ab}$ & $18,96 \mathrm{a}$ & $21,28 \mathrm{a}$ \\
\hline
\end{tabular}

Promedios con letras distintas en la misma columna indican diferencia significativa según la prueba de Duncan $(P \leq 0,05)$.

La concentración de $0,13 \%$ de $\mathrm{S}$ en la masa seca foliar de cebollín, deficiente de este elemento (tabla 2), está muy cerca al valor mínimo de $0,10 \%$ que deben contener a hortalizas para evitar síntomas de deficiencia visibles (Bergmann, 1993). Este autor describe que en el caso de una deficiencia de azufre, el sulfato de las hojas maduras no es suficiente para un crecimiento normal de la planta, generando hojas jóvenes más pequeñas debido a una carencia de proteínas y clorofilas.

Para la variable longitud de hoja no se encontraron diferencias significativas entre los tratamientos en el primer corte. En el segundo corte se puede observar los valores más bajos $(P \leq 0,05)$ en los tratamientos deficientes en $\mathrm{P}$ y $\mathrm{N}$, debido a que estos elementos interfieren en los procesos fotosintéticos y de elongación celular (Salisbury y Ross, 1994). La falta del $\mathrm{N}$ debilita la planta, se desarrolla poco y las hojas permanecen pequeñas (Navarro y Navarro, 2000), por lo cual, Bonilla (2008) describe este síntoma como enanismo (figura 1).

El P se manifestó de nuevo como uno de los elementos más importantes para el desarrollo de la planta de cebollín, especialmente en el segundo corte, donde redujo drásticamente el desarrollo foliar, lo que explica Bender (1993) como un crecimiento muy lento de la planta y un retardo en su madurez (Bonilla, 2008).

\section{Masa seca de raíz y hojas}

Entre el primer y segundo corte la masa seca, acumulada en raíz y hojas, aumentó considerablemente (tabla 5).

Especialmente la ausencia del P en la solución nutritiva provocó una baja producción de biomasa radical que también perjudicó la acumulación de masa seca foliar (tabla 5) y de la planta en total (figura 2). Este resultado refleja la importancia del fósforo para la formación del sistema radical (Gómez, 2006), siendo los fosfatos esenciales para la división celular y para el desarrollo de los tejidos meristemáticos (Wild y Jones, 1992) y además subraya el papel clave del $\mathrm{P}$ en el metabolismo energético de la planta (Bonilla, 2008) que se manifiesta en una formación deficiente de los órganos del cebollín. Las otras deficiencias no afectaron significativamente la acumulación de MS en la raíz del cebollín.

También, la deficiencia de P mostró los valores más bajos en MS foliar (tabla 5), que se redujo en un $67,87 \%$ con respecto al testigo, esto demuestra nuevamente que el $\mathrm{P}$ es esencial para la transferencia energética en la planta y afecta, según Bender (1993), todos los aspectos del crecimiento de las aliáceas y concuerda con lo que se encontró para cebollín (figura 2). 
Tabla 5. Efecto de la deficiencia de N, P, K, Ca, Mg y S sobre la masa seca en hojas y raíz en la planta de cebollín en el primer ( $69 \mathrm{dds}$ ) y segundo corte ( $110 \mathrm{dds})$.

\begin{tabular}{|l|c|c|c|c|}
\multirow{2}{*}{ Tratamiento } & \multicolumn{2}{|c|}{ Masa seca raíz $(\mathrm{g})$} & \multicolumn{2}{c|}{ Masa seca hoja $(\mathrm{g})$} \\
\cline { 2 - 5 } & Primer corte & Segundo corte & Primer corte & Segundo corte \\
\hline Testigo & $0,034 \mathrm{ab}$ & $0,149 \mathrm{a}$ & $0,077 \mathrm{a}$ & $0,194 \mathrm{~b}$ \\
\hline N 25\% & $0,039 \mathrm{a}$ & $0,148 \mathrm{a}$ & $0,061 \mathrm{a}$ & $0,161 \mathrm{bc}$ \\
\hline -P & $0,016 \mathrm{~b}$ & $0,069 \mathrm{~b}$ & $0,036 \mathrm{~b}$ & $0,063 \mathrm{c}$ \\
\hline -K & $0,031 \mathrm{ab}$ & $0,116 \mathrm{ab}$ & $0,069 \mathrm{ab}$ & $0,194 \mathrm{bc}$ \\
\hline -Ca & $0,023 \mathrm{ab}$ & $0,100 \mathrm{ab}$ & $0,058 \mathrm{ab}$ & $0,232 \mathrm{~b}$ \\
\hline -Mg & $0,018 \mathrm{~b}$ & $0,132 \mathrm{ab}$ & $0,045 \mathrm{ab}$ & $0,324 \mathrm{a}$ \\
\hline$-S$ & $0,022 \mathrm{ab}$ & $0,113 \mathrm{ab}$ & $0,051 \mathrm{ab}$ & $0,209 \mathrm{~b}$ \\
\hline
\end{tabular}

Promedios con letras distintas en la misma columna indican diferencia significativa según la prueba de Duncan $(P \leq 0,05)$.

En el segundo corte, la segunda producción más baja de la MS foliar fue mostrada por la aplicación del $\mathrm{N}$ al 25\%, sin mostrar diferencias significativas con el testigo (tabla 5). Resende y Souza (2001) encontraron en ajo que la deficiencia de $\mathrm{N}$ afecta la producción de masa seca y Bender (1993) afirma que una carencia de $\mathrm{N}$ en cebolla ocasiona plantas pequeñas, lo que se confirma en lo presentado en la figura 1. Lemaire et al. (2008) constatan que la reducción del contenido de $\mathrm{N}$ por unidad de área foliar (tabla 2) reduce la eficiencia en el uso de la radiación que, en consecuencia, afecta la tasa fotosintética.

La ausencia de $\mathrm{K}$ en la fertilización no afectó mucho la acumulación de MS en los cebollines de este tratamiento (figura 2), debido a que en el tejido foliar de estas plantas se aumentaron las concentraciones de N, Py S comparado con el testigo (tabla 2). El N optimiza la tasa de expansión de la hoja y así la intercepción de la radiación fotosintéticamente activa (Lemaire et al., 2008).

Similar a la deficiencia de K, la de Ca no afectó significativamente la producción de MS de la planta, esto se debe a que la ausencia de este elemento en el agua de riego fue compensada por una mayor acumulación de $\mathrm{N}, \mathrm{P}$ y $\mathrm{S}$ en el tejido foliar del cebollín. Tal vez, la poca reducción de este elemento en la hoja (13,7\%, tabla 2) generó condiciones que no fueron suficientemente extremas para que se mostrara una reducción de la biomasa (Bergmann, 1993). En especies del genero Aliaceae como la cebolla, la deficiencia de Ca es poco común, sin embargo, en el caso de una deficiencia de $\mathrm{Ca} 2+$ extrema las plantas no son capaces de sobrevivir y solamente en pocos casos pasan el estado de planta germinada (Bender, 1993).

La deficiencia absoluta de $\mathrm{Mg}$ en la solución nutritiva en el primer corte no tuvo diferencias con los demás tratamientos, mientras que en el segundo corte hubo un aumento significativo de la MS foliar, debido seguramente a las concentraciones incrementadas de N, P, Ca y S en este órgano, comparadas con el testigo (tabla 2), lo que se refleja en la tabla 5. Es conocido que el Mn, Zn y Ca pueden reemplazar el $\mathrm{Mg}$ en varias de sus funciones (Bergmann, 1993).

La deficiencia de azufre no produjo acumulación diferente $(P \leq 0,05)$ de MS en la planta comparada con el testigo (tabla 5), debido a la no reducción del contenido de este elemento en plantas sin S (tabla 2). Así mismo, Bender (1993) reporta que esta deficiencia no es común en aliáceas, como por ejemplo en la cebolla. 


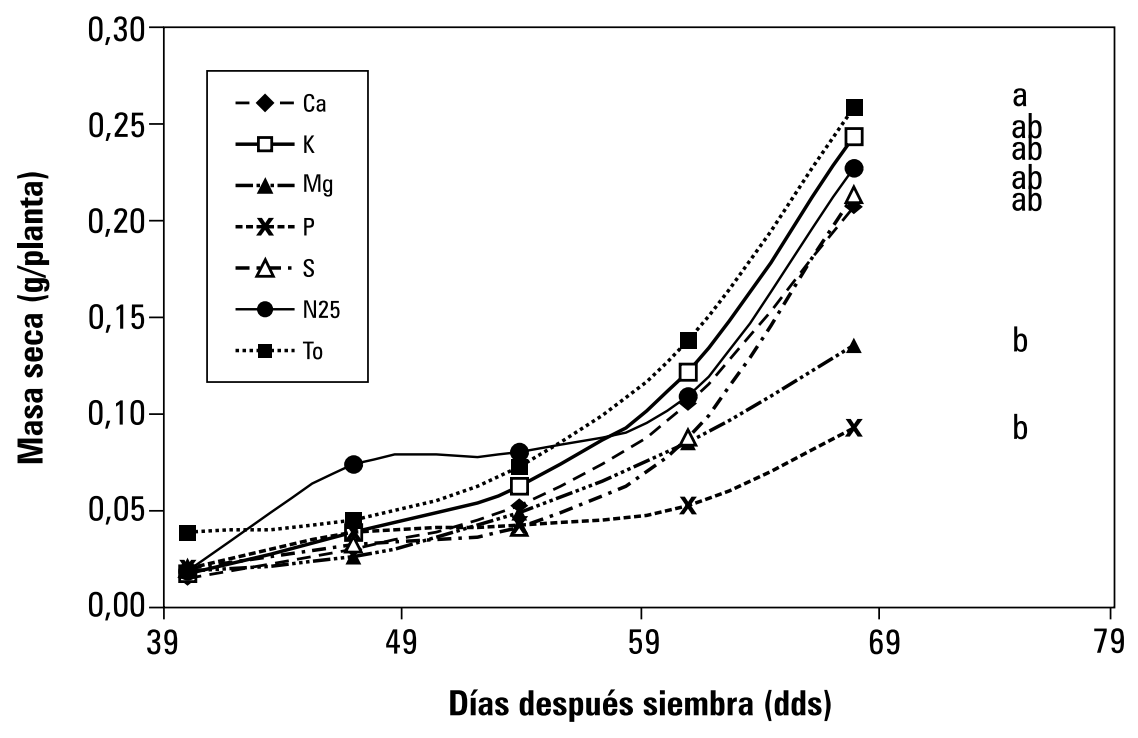

Figura 2. Efecto de la deficiencia de $N, P, K$ y $S$ sobre la acumulación de la masa seca total en cebollín en el primer corte ( $69 \mathrm{dds}$ ). Promedios con letras distintas indican diferencia significativa a los 69 días del primer ciclo según la prueba de Duncan $(P \leq 0,05)$.

\section{CONCLUSIONES}

- El estudio determinó que una nutrición deficiente en $\mathrm{P}$ y $\mathrm{N}$ afecta el crecimiento y desarrollo del cebollín, especialmente durante el segundo ciclo del cultivo

- La deficiencia de $\mathrm{P}$, que tiene funciones claves en el metabolismo energético de la planta, causó disminución notable en el área foliar y la acumulación de masa seca en hoja y raíz.

- Dentro de los seis elementos estudiados los más limitantes para el aumento de la bioma- sa seca de la planta, bajo las condiciones del experimento, fueron en un orden decreciente, en el primer corte: $\mathrm{P}>\mathrm{Mg}>\mathrm{S}>\mathrm{Ca}>\mathrm{N}>\mathrm{K}$ y en el segundo corte: $\mathrm{P}>\mathrm{N}>\mathrm{K}>\mathrm{S}>\mathrm{Ca}>\mathrm{Mg}$.

- Las deficiencias de $\mathrm{K}$ y Ca no afectaron significativamente el crecimiento de las plantas.

- La carencia del Mg aumentó el número y la biomasa de las hojas en el segundo corte del cebollín por el incremento de N, P, Ca y $\mathrm{S}$ en la masa seca foliar de este tratamiento.

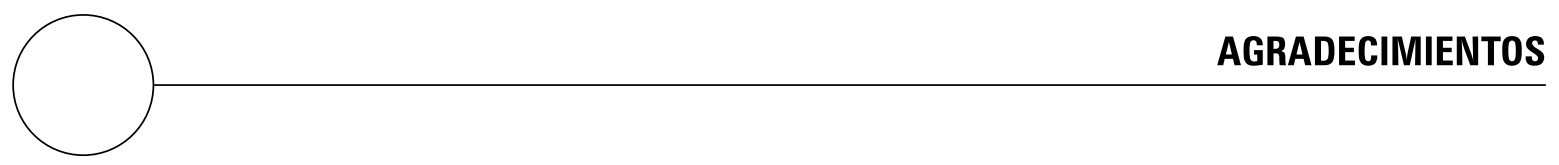

Los autores agradecen el apoyo para realizar este trabajo al Departamento de Agro de Brenntag Colombia S.A., Mosquera, especialmente a Hugo Bolhuis y Milton Galán. 


\section{REFERENCIAS BIBLIOGRÁFICAS}

Abello, J.; J. Clavijo y P. Barreno. 2006. Estudio preliminar de algunos descriptores fisiológicos en cinco hierbas aromáticas. pp. 13-15. En: Memorias Curso de Extensión: Últimas tendencias en hierbas aromáticas culinarias para exportación en fresco. Facultad de Agronomía, Universidad Nacional de Colombia, Bogotá.

Alves, C.; D. Alves; L.A. Fernandes; E.R. Martins; I.G. Souza; R.A. Sampaio y P.S. Lopes. 2007. Nutrição mineral da fava d'anta. Hortic. Brás. 25(1), 24-28.

Barreño, P. 2006. Hierbas aromáticas culinarias para exportación en fresco manejo agronómico, producción y costos. pp. 65-72. En: Memorias Curso de Extensión: Últimas tendencias en hierbas aromáticas culinarias para exportación en fresco. Facultad de Agronomía, Universidad Nacional de Colombia, Bogotá.

Barreño, P. y J. Clavijo. 2006. Hierbas aromáticas culinarias para exportación en fresco. pp. 7-9. Memorias Curso de Extensión: Últimas tendencias en hierbas aromáticas culinarias para exportación en fresco. Facultad de Agronomía, Universidad Nacional de Colombia, Bogotá.

Bender, D.A. 1993. Onions. pp. 131-135. En: Bennett, W.F. (ed.). Nutrient deficiencies and toxicities in crop plants. APS Press, St. Paul, MN.

Bergmann, W. 1993. Ernährungsstörungen bei Kulturpflanzen. $3 a$ ed. Gustav Fischer Verlag, Jena, Alemania.

Bonilla, I. 2008. Introducción a la nutrición mineral de las plantas. Los elementos minerales. pp. 102-121. En: Azcón-Bieto, A. y M. Talón. (eds.). Fundamentos de la fisiología vegetal. McGraw-Hill Interamericana, Madrid.

Castro, H.E. 1998. Capítulo 4: Hortalizas, producción y fertilización de hortalizas en Colombia. pp., 170194. En: Guerrero, R. (ed.). Fertilización en cultivos de clima frío. Monómeros Colombo Venezolanos S.A., Colombia.

Castro, J.V.; L.B. Pfaffenbach; C.R.L Carvalho y C.J. Rossetto. 2003. Efecto del empaque plástico sobre la vida de anaquel del mango 'Keitt'. Rev. Iberoamer. Tecnol. Poscos. 5(1), 33-37.

Clavijo, J. 2006. Fisiología de la producción de hierbas aromáticas. pp. 10-12. En: Memorias Curso de Ex- tensión: Últimas tendencias en hierbas aromáticas culinarias para exportación en fresco. Facultad de Agronomía, Universidad Nacional de Colombia, Bogotá.

Delahaut, K.A. y A.C. Newenhouse. 2003. Growing onions, garlic, leeks, and other Allium in Wisconsin: A guide for fresh-market growers. Cooperative Extension, University of Wisconsin, Madison, WI.

Epstein, E. y A.J. Bloom. 2005. Mineral nutrition of plants: principles and perspectivas. 2 a ed. Sinauer Associates Publishers, Sunderland, MA.

Gómez, M.I. 2006. Manual técnico de fertilización de cultivos. Microfertisa, Produmedios, Bogotá.

Jones, J.B. 1998. Manual of plant nutrition. CRC Press, Boca Raton, FL.

Le Bot, J.; S. Adamowicz y P. Robín. 1998. Modeling plant nutrition of horticultural crops: a review. Scientia Hort. 74(1-2), 47-82.

Lemaire, G.; E. Oosterom; M.H. Jeuffroy; F. Gastal y A. Massignam. 2008. Crop species present different qualitative types of response to $\mathrm{N}$ deficiency during their vegetative growth. Field Crops Res. 105(1), 253-265.

Liu, S.; H. He; G. Feng y Q. Chen. 2009. Effect of nitrogen and sulfur interaction on growth and pungency of different pseudostem types of Chinese spring onion (Allium fistulosum L.). Scientia Hort. 121, en imprenta.

Marschner, H. 2002. Mineral nutrition of higher plants. Academic Press, Londres.

Martínez, F.E.; J. Sarmiento; G. Fischer y F. Jiménez. 2009. Síntomas de deficiencia de macronutrientes y boro en plantas de uchuva (Physalis peruviana L.). Agron. Colomb. 27(2), en imprenta.

Navarro, S. y G. Navarro. 2000. Química agrícola. Ediciones Mundi-Prensa, Madrid.

Öpik, H. y S. Rolfe. 2005. The physiology of flowering plants. Cambridge University Press, Cambridge, UK.

Pôrto, D,R.; A.B. Cecílio; A. May y J.C. Barbosa. 2006. Acúmulo de macronutrientes pela cebola 'Optima' 
estabelecida por semeadura directa. Hortic. Bras. 24(4), 470-475.

Ramírez, C. 2006. Nutrición mineral y producción vegetal. pp. 22-30. En: Memorias Curso de Extensión: Últimas tendencias en hierbas aromáticas culinarias para exportación en fresco. Facultad de Agronomía, Universidad Nacional de Colombia, Bogotá.

Resende, G.My R.J. Souza. 2001. Doses e épocas de aplicação de nitrogênio sobre a productividade e características comerciais de alho. Hortic. Brás. 19(2), $126-129$

Sabino, H.; J. Lavres y M. Ferreira. 2007. Azufre como nutriente y agente de defensa contra plagas y enfermedades. Informaciones Agronómicas. International Plant Nutrition Institute 65(1), 1-4.
Salisbury, F.B. y C.W. Ross. 2000. Fisiología de las plantas. Editorial Paraninfo Thomson Learning, Madrid.

Steinmetz, K.A. y J.D. Potter. 1996. Vegetables, fruit, and cancer prevention: A review. J. Amer. Diet. Assoc. 96(1), 1027-1039.

Taiz, L. y E. Zeiger. 2006. Plant physiology, 4a ed. Sinauer Associates, Sunderland, MA.

Wild, A. y L.H.P. Jones. 1992. Nutrición mineral de las plantas cultivadas. pp. 73-119. En: Wild, A. (ed.) Condiciones del suelo y desarrollo de las plantas según Russel. Ediciones Mundi-Prensa, Madrid.

You, W.C.; W.J. Blot; Y.S. Chang; A. Ershow; Z.T. Yang; Q. An; B.E. Henderson; J.F. Fraumeni y T.-G. Wang. 1989. Allium vegetables and the reduced risk of stomach cancer. J. Natl. Cancer Inst. 81, 162-164. 